\title{
Aktuelle Konzepte zum mikrobiologischen Nachweis von Atemwegserregern
}

\author{
Current Concepts for the Microbiological Detection of Airway Pathogens
}

Autoren

Institute
G. Höffken ${ }^{1,3}$, M. Kolditz ${ }^{1}$, M. Halank ${ }^{1}$, C. Lück² ${ }^{2}$ D. Koschel ${ }^{3}$

Medizinische Klinik 1/Pneumologie des Universitätsklinikums Carl Gustav Carus (Leiter: Prof. Dr. med. G. Ehninger)

Institut für Klinische Mikrobiologie der Technischen Universität Dresden (Leiter: Prof. Dr. med. E. Jacobs)

Abteilung Innere Medizin/Pneumologie des Fachkrankenhauses Coswig, Zentrum für Pneumologie, Allergologie, Beatmungsmedizin, Thorax- und Gefäßchirurgie, Coswig (Leiter: Prof. Dr. med. G. Höffken) eingereicht 17.7.2009

akzeptiert 4.8.2009

Bibliografie

DOI http://dx.doi.org/

10.1055/s-0029-1215132

Online-Publikation: 16. 11. 2009

Pneumologie 2010; 64:

184-193 @ Georg Thieme

Verlag KG Stuttgart · New York

ISSN 0934-8387

\section{Korrespondenzadresse \\ Prof. Dr. Gert Höffken}

Medizinische Klinik 1/

Pneumologie des Universitäts-

klinikums Carl Gustav Carus

Abteilung Innere Medizin/

Pneumologie des Fachkranken-

hauses Coswig

Fetscherstr. 74

01309 Dresden

gert.hoeffken@uniklinikum-

dresden.de

\section{Serienherausgeber \\ B. Schaaf, Dortmund \\ G. Rohde, Bochum}

\section{Zusammenfassung \\ $\nabla$}

Die Häufigkeit und das Spektrum bakterieller Erreger bei Atemwegsinfektionen sind von einer Vielzahl von Variablen abhängig. Die Epidemiologie wird entscheidend durch die Art der zugrunde liegenden Atemwegsinfektionen, die Abwehrlage des Patienten, geographische und saisonale Faktoren und den Umfang und die Auswahl der eingesetzten diagnostischen Methoden beeinflusst. Darüber hinaus ist die klinische Bedeutung eines positiven Erregernachweises aus respiratorischen Sekreten unsicher, da es sich um unsterile oder kontaminierte Materialien handelt. Die ätiologische Relevanz erschließt sich somit erst aus der synoptischen Beurteilung der mikrobiologischen und klinischen Daten, wobei der Einschätzung einer Immunsuppression oder lokalen Abwehrstörung eines Patienten hierbei eine wichtige Rolle zukommt.

\section{Abstract \\ $\nabla$}

The frequency and range of bacterial pathogens in respiratory tract infections depends on a variety of variables. The aetiology is influenced by the type of respiratory tract infection (acute bronchitis, acute exacerbation of chronic bronchitis, community-acquired pneumonia, nosocomial pneumonia), geographic and seasonal factors, and by the diagnostic methods implemented to identify the pathogens. Furthermore, there exists a considerable uncertainty concerning the clinical relevance of positive bacteriological findings in respiratory secretions because they are retrieved from non-sterile or contaminated environments. To differentiate infection from contamination, both microbiological and clinical findings have to be assessed, which might be difficult in the setting of systemic or local immunosuppression.

\section{Einleitung \\ $\nabla$}

Im Folgenden werden die wichtigsten Erreger von Infektionen der Atemwege sowie mikrobiologische Methoden und deren Bewertung zu ihrer Diagnostik dargestellt. Keine Berücksichtigung in der vorliegenden Zusammenfassung finden Infektionserreger beim immunsupprimierten Patienten und bei Patienten mit zystischer Fibose. Die Ausführungen basieren weitgehend auf der „S3Leitlinie der Paul-Ehrlich-Gesellschaft für Chemotherapie, der Deutschen Gesellschaft für Pneumologie, der Deutschen Gesellschaft für Infektiologie und vom Kompetenznetzwerk CAPNETZ zu Epidemiologie, Diagnostik, antimikrobieller Therapie und Management von erwachsenen Patienten mit ambulant erworbenen tiefen Atemwegsinfektionen (akute Bronchitis, akute Exazerbation einer chronischen Bronchitis, Influenza und andere respiratorische Virusinfektionen) sowie ambulant erworbener Pneumonie“ [1].

\section{Streptococcus pneumoniae}

S. pneumoniae sind grampositive Diplokokken, die bei $10-20 \%$ der gesunden Bevölkerung in der physiologischen oropharyngealen Flora als kolonisierende Bakterien nachgewiesen werden können. Sie haben bei Atemwegsinfektionen eine herausragende Bedeutung: Pneumokokken sind die wobenen Pneumonie [2] und werden auch bei der frühen (innerhalb der ersten 5-7 Tage nach stationärer Aufnahme) nosokomialen Pneumonie und bei der akuten Exazerbation einer chronischen Bronchitis regelmäßig isoliert. mit Abstand häufigsten Erreger der ambulant er- 
Tab. 1 Übersicht über diagnostische Verfahren von Atemwegserregern I.

\begin{tabular}{|c|c|c|c|c|c|c|}
\hline Erreger & Mikroskopie* & Kultur** & $\begin{array}{l}\text { Nukleinsäure- } \\
\text { amplifikations- } \\
\text { verfahren }\end{array}$ & $\begin{array}{l}\text { Antigen- } \\
\text { Nachweis }\end{array}$ & Serologie & Sonstiges \\
\hline S. pneumoniae & $\begin{array}{l}\text { Grampräparat } \\
\text { (grampositiv) }\end{array}$ & $\begin{array}{l}\text { Standard- } \\
\text { verfahren }\end{array}$ & n. v. ${ }^{* * *}$ & $\begin{array}{l}\text { Pneumokokken- } \\
\text { Antigentest } \\
\text { Sens. } 50-80 \% \\
\text { Spez. >90\% }\end{array}$ & n.v. & $\begin{array}{l}\text { häufigster Atemwegs- } \\
\text { erreger bei AECOPD, } \\
\text { CAP, frühe NAP**** }\end{array}$ \\
\hline H. influenzae & $\begin{array}{l}\text { Grampräparat } \\
\text { (gramnegativ) }\end{array}$ & $\begin{array}{l}\text { Standard- } \\
\text { verfahren }\end{array}$ & n. v. & n.v. & n.v. & $\begin{array}{l}\text { häufiger Erreger bei } \\
\text { AECOPD, selten bei } \\
\text { CAP, frühe NAP**** }\end{array}$ \\
\hline M. pneumoniae & n.v. & n.v. & $\begin{array}{l}\text { gute Genauigkeit, } \\
\text { nicht validiert }\end{array}$ & n.v. & $\begin{array}{l}\text { IgM-Nachweis } \\
\text { Sens. }>90 \% \\
\text { Spez. } 100 \%\end{array}$ & $\begin{array}{l}\text { IgM bei protrahierter } \\
\text { Symptomatik einer } \\
\text { CAP; < } 10 \% \text { bei CAP }\end{array}$ \\
\hline L. pneumophila & n.v. & $\begin{array}{l}\text { Spezialnähr- } \\
\text { böden, } \\
\text { Sens. } 10-80 \% \\
\text { Spez. } 100 \%\end{array}$ & $\begin{array}{l}\text { Real-time PCR } \\
\text { Sens. }>90 \% \\
\text { Spez. }>90 \%\end{array}$ & $\begin{array}{l}\text { Legionella-Ag } \\
\text { Test } \\
\text { Sens. } 75-90 \% \\
\text { Spez. >99\% }\end{array}$ & $\begin{array}{l}\text { nicht empfohlen, } \\
\text { da sowohl falsch- } \\
\text { positiv als auch } \\
\text {-negativ }\end{array}$ & $\begin{array}{l}\text { überwiegend Pneu- } \\
\text { monie-Erreger (außer } \\
\text { bei Pontiac-Fieber), } \\
<10 \% \text { bei CAP }\end{array}$ \\
\hline C. pneumoniae & n. v. & n.v. & $\begin{array}{l}\text { gute Genauigkeit, } \\
\text { nicht validiert }\end{array}$ & $\begin{array}{l}\text { keine Routine- } \\
\text { diagnostik }\end{array}$ & nicht empfohlen & $\begin{array}{l}\text { seltener Erreger bei } \\
\text { Atemwegsinfektio- } \\
\text { nen, Bedeutung über- } \\
\text { schätzt }\end{array}$ \\
\hline
\end{tabular}

\section{Diagnostische Verfahren}

Zum Nachweis einer S. pneumoniae-Infektion der Atemwege stehen folgende Verfahren zur Verfügung:

- Beurteilung des Grampräparates aus Proben der unteren Atemwege: Sensitivität und Spezifität sind abhängig von der Qualität der untersuchten Probe, der Transportzeit bis zur Untersuchung und der Erfahrung des Untersuchers; sie betragen bis zu ca. 80\%. Ein Vorteil der Untersuchung liegt im raschen Vorliegen des Testergebnisses.

- Kulturelle Anzucht von S. pneumoniae aus Proben der unteren Atemwege, aus Blutkulturen und Pleuraflüssigkeit mittels mikrobiologischer Standardverfahren: Der Nachweis aus sterilen Körperflüssigkeiten ist für eine Infektion beweisend. Die Sensitivität der Kultur aus Blut oder Pleurapunktat wird mit $10-30 \%$ angegeben.

- Pneumokokken-Antigentest im Urin (Binax NOW): Dieser Test, dessen Ergebnis innerhalb von weniger als 30 Minuten vorliegt, ist ein immunchromatografischer Membrantest, der das Pneumokokken-Zellwand-Polysaccharid (C-Substanz, nicht identisch mit dem Kapselpolysaccharid) nachweist. Das Antigen ist bei allen Serotypen von S. pneumoniae, aber auch bei S. oralis und S. mitis vorhanden [3].

Der Test hat, verglichen mit konventionellen diagnostischen Methoden, bei Erwachsenen eine Sensitivität von 50 bis $80 \%$ sowie eine Spezifität von ca. 90\% [4]. Wenn als Goldstandard eine „positive Pneumokokkenkultur in respiratorischen Sekreten“ zum Nachweis einer Pneumonie durch S. pneumoniae definiert wird, beträgt die Sensitivität allerdings nur 54\% [5]. Bei allen publizierten Angaben zur Sensitivität ist zu beachten, dass als „Goldstandard“ immer die verfügbaren klassischen Methoden herangezogen werden. Da diese nie $100 \%$ ig sensitiv sind, muss ein Teil der „falsch-positiven“ Ergebnisse als „richtig“ eingestuft werden. Die nicht ausreichende diagnostische Genauigkeit des Tests schließt daher bei negativem Testergebnis eine Pneumonie durch S. pneumoniae nicht aus bzw. bei einem positiven Ergebnis kann, wenn auch selten, ein falsch-positives Resultat vorliegen. Der Test wird daher zur Routinediagnostik bei ambulant erworbener Pneumo- nie, bei nosokomialer Pneumonie bzw. bei akuter Exazerbation einer chronischen Bronchitis nicht generell empfohlen. Dieses Vorgehen hat allerdings nur dann Gültigkeit, wenn die empirische Initialtherapie dieser Infektionen ein Pneumokokken-wirksames Antibiotikum beinhaltet [1,6,7]. Der Test kann dagegen eingesetzt werden, wenn bereits eine Therapie begonnen wurde, und so zur diagnostischen Klärung beitragen.

\section{Empfehlung zur Diagnostik ( 0 Tab. 1)}

Eine Sputumuntersuchung auf respiratorische Erreger inkl. $S$. pneumoniae mit Anfertigung eines Gram-Präparates und einer konventionellen Kultur sollte nur durchgeführt werden, wenn folgende Kriterien erfüllt sind:

- umgehende Bearbeitung der Proben innerhalb von 2 Stunden im Labor sowie

- ausreichende mikroskopische Sputumqualität (>25 Granulozyten und < 10 Plattenepithelien pro Gesichtsfeld bei 100-facher Vergrößerung) [1].

Sie wird empfohlen

- bei hospitalisierten Patienten mit akuter Exazerbation einer chronischen Bronchitis,

- bei Patienten mit ambulant-erworbener Pneumonie (im ambulanten Bereich oder hospitalisiert) mit Verdacht auf resistente oder ungewöhnliche Erreger,

- bei Patienten mit nosokomialer Pneumonie.

Die Entnahme von Blutkulturen wird empfohlen

- bei Patienten mit schwerer ambulant erworbener Pneumonie, - bei Patienten mit nosokomialer Pneumonie.

Der Pneumokokken-Antigentest bedarf einer kritischen Interpretation und wird im Rahmen der Routinediagnostik nicht generell empfohlen.

\section{Kontaktadresse}

Nationales Referenzzentrum für Streptokokken/Pneumokokken: Ansprechpartner Prof. Rudolf Lütticken, Institut für Mikrobiologie RWTH Aachen, Pauwelstr. 30, 52057 Aachen, Tel.: 02418089510, www.streptococcus.de. 


\section{Haemophilus influenzae}

$\nabla$

H. influenzae sind kleine, pleomorphe, gramnegative Stäbchen. Sie sind ein Haupterreger bei bakteriellen Exazerbationen einer chronischen Bronchitis und spielen eine Rolle als Erreger der ambulant erworbenen Pneumonie (Häufigkeit nach den CAPNETZ-Daten $<10 \%$ ) [2] sowie auch der frühen nosokomialen Pneumonie.

\section{Diagnostische Verfahren}

Zum Nachweis von $\mathrm{H}$. influenzae stehen die Beurteilung des Grampräparates aus Proben der unteren Atemwege und die kulturelle Anzucht aus Proben der unteren Atemwege, Blut und Pleuraflüssigkeit zur Verfügung.

\section{Empfehlung zur Diagnostik ( 0 Tab. 1)}

Die Empfehlungen zur mikrobiologischen Diagnostik tiefer Atemwegsmaterialien entsprechen denen bei Pneumokokken.

\section{Kontaktadresse}

Konsiliarlabor Haemophilus, Ansprechpartner Prof. Mathias Frosch, Institut für Hygiene und Mikrobiologie Würzburg, Josef-Schneider-Str. 2, 97080 Würzburg, Tel.: 0931-20146161, www.haemophilus-online.de.

\section{Mycoplasma pneumoniae \\ $\nabla$}

M. pneumoniae ist ein zellwandloses Bakterium, das sich auf Nährmedien nur schwer anzüchten lässt. Es ist ein Erreger der ambulant erworbenen Pneumonie (Häufigkeit nach den CAPNETZ Daten < 10\%) [2]. Mykoplasmen haben eine zu vernachlässigende Bedeutung als Pathogen der nosokomialen Pneumonie. Ihre klinische Relevanz bei der akuten Exazerbation einer chronischen Bronchitis ist unsicher und wird eher als unbedeutend eingeschätzt $[1,9]$.

\section{Diagnostische Verfahren \\ Kultur}

Aufgrund des technischen Aufwandes, der Dauer und der im Vergleich zu molekularbiologischen Methoden geringeren Nachweisrate aus respiratorischen Sekreten spielt die Kultur für die klinische Diagnostik einer M.-pneumoniae-Infektion keine Rolle $[1,8]$.

\section{Serologie}

Für die serologische Diagnostik stehen folgende Testverfahren zur Verfügung:

- die Komplementbindungsreaktion, die keine ausreichende Sensitivität und Spezifität aufweist [10] und nicht mehr eingesetzt werden sollte

- der Nachweis von Kälte-Agglutininen, der mit dem Schweregrad der Erkrankung korreliert, aber eine ungenügende Genauigkeit besitzt [11]

- der Nachweis von IgG-, IgM- und IgA-Antikörpern mittels Enzymimmunoassay (EIA), wobei verschiedene kommerzielle EIA-Testverfahren zur Verfügung stehen. Da IgM-Antikörper häufig nur bei Kindern und jungen Erwachsenen nach Erstkontakt mit M. pneumoniae auftreten, schließt ein negativer IgM-Nachweis eine M.-pneumoniae-Infektion, insbesondere bei älteren Erwachsenen, nicht aus. Bei länger bestehender (>12 Tage) klinischer Symptomatik einer ambulant erworbenen Pneumonie ist die Sensitivität und Spezifität eines IgM-
Nachweises signifikant höher und kann daher zur Diagnostik empfohlen werden [12]. Die IgA-Bestimmung weist bei Erwachsenen eine höhere Sensitivität, wegen der langen Persistenz der IgA-Antikörper nach Infektion aber eine ungenügende Spezifität auf [13]. Zu beachten sind falsch-positive Ergebnisse infolge Kreuzreaktionen mit diversen Erregern, u.a. Legionella spp., Chlamydophila spp. und S. pneumoniae, die bei verschiedenen Testverfahren unterschiedlich häufig beschrieben werden [14].

\section{Amplifikationsverfahren}

Aufgrund der schwierigen Kultivierbarkeit von M. pneumoniae wurden verschiedene Amplifikationsverfahren (z. B. PolymeraseKettenreaktion [PCR]) zum Nachweis dieses Erregers entwickelt, die im Vergleich zur konventionellen serologischen Diagnostik eine deutlich verbesserte Sensitivität und Spezifität besitzen [8]. Ein genereller Nachteil dieser Verfahren sind falsch-positive Nachweise, da M. pneumoniae während der Inkubationszeit oder nach Erkrankung den Respirationstrakt zeitweise besiedeln kann, ohne an der aktuellen Erkrankung beteiligt zu sein. Studien belegen, dass die diagnostische Ausbeute durch Einsatz der Real-time-PCR in der Diagnostik von ambulant erworbenen Pneumonien im Vergleich zur konventionellen Diagnostik gesteigert werden kann [14]. Aufgrund nicht einheitlicher Standardisierung der Präanalytik und Analytik, aber auch der Kosten und fehlender Kosten-Effizienz-Untersuchungen sollten die Amplifikationsverfahren noch nicht in der Routinediagnostik eingesetzt werden [1].

\section{Empfehlung zur Diagnostik ( 0 Tab. 1):}

Serologische Diagnostik einer M.-pneumoniae-Infektion (IgMNachweis):

- optional bei mittelschwerer ambulant erworbener Pneumonie mit verlängerter klinischer Symptomatik (>12 Tage) bzw. Therapieversagen nach Betalaktam-Therapie

- keine Empfehlung bei allen anderen Formen einer ambulant erworbenen Pneumonie, nosokomialer Pneumonie, akuter Bronchitis und akuter Exazerbation einer chronischen Bronchitis

Nukleinsäure-Amplifikationsverfahren (Real-time-PCR) sind als experimentelle Verfahren mit noch nicht ausreichend gesicherter Evidenz für den Routineeinsatz einzuschätzen.

\section{Kontaktadresse}

Konsiliarlabor für Deutschland: Institut für Klinische Mikrobiologie der Technischen Universität Dresden, Ansprechpartner Prof. Dr. med. Enno Jacobs, Tel.: 0351-4596550; enno.jacobs@tu-dresden.de, www.tu-dresden.de/medimmh/konsiliarlabore.html.

\section{Legionella spp. \\ $\nabla$}

Legionella spp. sind intrazelluläre Bakterien, als Krankheitserreger in Europa kommt überwiegend L. pneumophila vor. L. pneumophila ist ein Erreger der ambulant erworbenen Pneumonie, wobei nicht nur schwere, sondern auch leichte bis mittelschwere Verlaufsformen auftreten können. Als Erreger einer nosokomialen Pneumonie kommen Legionellen nur sporadisch vor, eventuell im Rahmen von Kleinraumendemien durch kontaminiertes Wasser. Bei akuter Bronchitis und akuter Exazerbation einer chronischen Bronchitis haben Legionellen keine ätiologische Bedeutung. Die Bedeutung des Pontiac-Fiebers als nicht pneumonische Verlaufsform ist nicht ausreichend untersucht. 
Da Legionellen den Respirationstrakt nicht kolonisieren, ist der Nachweis aus respiratorischen Sekreten in aller Regel gleichbedeutend mit einer Infektion. Aufgrund des ubiquitären Vorkommens in der Umwelt sind Kontaminationen zu berücksichtigen.

\section{Diagnostische Verfahren}

Zur Diagnostik stehen verschiedene Verfahren zur Verfügung, wobei dem Nachweis von Legionella-Antigen im Urin die größte klinische Bedeutung zukommt.

\section{Antigentest}

Sowohl mit dem Enzym-Immuno-Assay als auch mit dem immunchromatografischen Schnelltest können nur L. pneumophila der Serogruppe 1 mit ausreichender Empfindlichkeit nachgewiesen werden, die in Deutschland die klinisch bedeutsamste Legionella-Spezies darstellen [16]. Die Testsysteme besitzen eine außerordentlich hohe Spezifität (>99\%), das heißt falsch-positive Ergebnisse kommen praktisch nicht vor. Die Sensitivität variiert je nach Ort der Entstehung der Infektion: Bei Reise-assoziierten Legionella-Infektionen liegt die Genauigkeit über 90\%, bei heimischen Legionellosen bei über 75\% [17]. Die Sensitivität der Antigennachweise kann etwas gesteigert werden, wenn der Urin durch Ultrafiltration konzentriert wird. Ein negativer Antigennachweis schließt eine Legionellose nicht aus; daher sind bei entsprechendem klinischem Verdacht Mehrfachuntersuchungen indiziert.

\section{Nukleinsäureamplifikationsverfahren}

Die konventionellen PCR-Techniken, die an Untersuchungsproben wie bronchoalveolärer Lavage, Trachealsekret und Sputum eingesetzt werden können, besitzen eine außerordentlich hohe diagnostische Ausbeute und liefern bessere Ergebnisse als die Kulturverfahren [18]. Sie sind für Materialien, die nicht aus den Atemwegen stammen (Serum, Urin), noch nicht ausreichend untersucht. Die Real-time-PCR weist eine Sensitivität und Spezifität von über $90 \%$ auf und liefert das Ergebnis innerhalb von wenigen Stunden. Grundsätzlich gilt auch für die molekularbiologischen Methoden in der Diagnostik einer Legionellose, dass die Verfahren derzeit nicht in der Routinediagnostik eingesetzt werden sollten, da sie nur in Speziallabors ausreichend validiert sind. Diese sollten zwingend an den in Deutschland verfügbaren Ringversuchen teilnehmen.

Kultur

Geeignete Materialien für die Anzüchtung von Legionellen aus dem Respirationstrakt sind Trachealsekret, bronchoalveoläre Lavage und Lungengewebe, wobei Spezialnährböden (Zusatz von Holzkohle und Hefeextrakt [BCYE-Agar]) und verlängerte Bebrütungszeiten von bis zu 7 Tagen notwendig sind. Der klinische Verdacht auf eine Legionellose sollte daher dem Labor mit der Einsendung der Proben mitgeteilt werden. Die Sensitivität ist sehr variabel (zwischen $<10 \%$ und $80 \%$ ), die Spezifität beträgt $100 \%$ [19]. Ein Vorteil der Anzüchtung besteht darin, dass bei Verdacht auf Kontamination bzw. Infektion durch Wasser ein molekularer Vergleich der Legionella-Spezies aus klinischen Proben und Umgebungsmaterialien möglich ist [1].

\section{Direkte Immunfluoreszenz}

Die Sensitivität der direkten Immunfluoreszenz aus respiratorischen Proben ist mit 25-66\% unzureichend, eine Legionellose kann damit keinesfalls ausgeschlossen werden. Das Verfahren ist stark von der Erfahrung des jeweiligen Labors abhängig. Im Un- terschied zum Urinantigentest werden aber alle Serotypen von L. pneumophila mit der gleichen Sensitivität detektiert. Dies spielt insbesondere bei nosokomialen Infektionen eine Rolle. Für ambulant erworbene und Reise-assoziierte Erkrankungen ist der Antigentest im Urin besser geeignet [20].

\section{Serologie}

Mittels indirektem Immunfluoreszenztest können aus dem Serum IgG-, IgA- und IgM-Antikörper nachgewiesen werden. Ein vierfacher Titeranstieg bei einem Serumpaar im Abstand von 10 bis 14 Tagen gilt als Beweis für eine Infektion. Allerdings gibt es wichtige Einschränkungen in der klinischen Anwendung, die die unzureichende Sensitivität und Spezifität des Immunfluoreszenztestes erklären. Im Rahmen einer akuten Infektion durch $L$. pneumophila kann die Antikörperbildung verzögert sein (signifikante Titeranstiege erst 3 bis 6 Wochen post infectionem) bzw. bei einem Teil der Patienten komplett unterbleiben $[21,22]$. Falsch-positive Resultate können durch persistierende Antikörpertiter über Jahre nach einer Infektion (subklinisch oder Pontiac-Fieber) entstehen, was die Interpretation als Ausdruck einer akuten Infektion erheblich erschwert [23]. Weiterhin sind Kreuzreaktionen mit anderen Bakterienspezies, wie zum Beispiel Coxiella burnetii, Campylobacter spp. und Pseudomonas spp. beschrieben [24]. Die routinemäßige Legionella-Antikörperbestimmung wird daher in der Diagnostik von akuten Atemwegsinfektionen nicht empfohlen.

\section{Empfehlung zur Diagnostik ( 0 Tab. 1)}

Das Verfahren der Wahl ist der Antigentest im Urin. Als experimentelle Verfahren mit noch nicht ausreichend gesicherter Evidenz für den Routineeinsatz sind die Nukleinsäure-Amplifikationsverfahren (konventionelle PCR, Real-time-PCR) einzuschätzen.

Eine Diagnostik auf Legionellen wird empfohlen:

optional

- bei mittelschwerer CAP (hospitalisiert)

- bei therapierefraktärer nosokomialer Pneumonie

obligatorisch

- bei schwerer CAP

- bei therapierefraktärer CAP

keine Empfehlung

- bei akuter Bronchitis, akuter Exazerbation einer chronischen Bronchitis

\section{Kontaktadresse}

Konsiliarlabor für Deutschland: Institut für Klinische Mikrobiologie der Technischen Universität Dresden (Prof. Dr. med. E. Jacobs), Ansprechpartner Dr. med. C. Lück, Tel.: 0351-4586580; christian. lueck@tu-dresden.de, www.tu-dresden.de/medimmh/konsiliarlabor_legionellen.html.

\section{Chlamydophila pneumoniae}

Chlamydophila pneumoniae (Chlamydien) sind intrazellulär wachsende Bakterien, die bei Kindern und Erwachsenen zu Infektionen im oberen und unteren Respirationstrakt führen. Es sind in aller Regel selbstlimitierende Erkrankungen, die möglicherweise als Schrittmacherinfektionen zu bakteriellen Superinfektionen prädisponieren [25]. Basierend auf Mikroimmunfluoreszenzverfahren wurde die Häufigkeit von Chlamydieninfektionen bei der ambulant erworbenen Pneumonie zwischen 5 und 
Tab. 2 Übersicht über diagnostische Verfahren von Atemwegserregern II.

\begin{tabular}{|c|c|c|c|c|c|c|}
\hline Erreger & Mikroskopie* & Kultur** & $\begin{array}{l}\text { Nukleinsäure- } \\
\text { amplifikations- } \\
\text { verfahren }\end{array}$ & $\begin{array}{l}\text { Antigen- } \\
\text { Nachweis }\end{array}$ & Serologie & Sonstiges \\
\hline C.psittacii & n. v. ${ }^{* * *}$ & keine Routine & $\begin{array}{l}\text { hochsensitiv, } \\
\text { nicht validiert }\end{array}$ & n.v. & $\begin{array}{l}\text { Referenzmethode } \\
\text { MIF }\end{array}$ & $\begin{array}{l}\text { anamnestische } \\
\text { Angaben bedeutsam }\end{array}$ \\
\hline C. burnetii & n.v. & keine Routine & nur Speziallabors & n.v. & $\begin{array}{l}\text { ausreichende } \\
\text { Genauigkeit }\end{array}$ & $\begin{array}{l}\text { anamnestische } \\
\text { Angaben bedeutsam }\end{array}$ \\
\hline P. aeruginosa & $\begin{array}{l}\text { Gramfärbung } \\
\text { (gramnegatives } \\
\text { Stäbchen) }\end{array}$ & $\begin{array}{l}\text { Standard- } \\
\text { verfahren }\end{array}$ & n.v. & n.v. & n.v. & $\begin{array}{l}\text { Risikofaktoren beach- } \\
\text { ten, Infektion versus } \\
\text { Kolonisation: CPIS- } \\
\text { Score; häufig bei Be- } \\
\text { atmungspneumonie }\end{array}$ \\
\hline $\begin{array}{l}\text { Nonfermenter } \\
\text { (außer P. aer.) }\end{array}$ & $\begin{array}{l}\text { Gramfärbung } \\
\text { (gramnegatives } \\
\text { Stäbchen) }\end{array}$ & $\begin{array}{l}\text { Standard- } \\
\text { verfahren }\end{array}$ & n.v. & n.v. & n.v. & $\begin{array}{l}\text { Risikofaktoren beach- } \\
\text { ten; bei Beatmungs- } \\
\text { pneumonie }\end{array}$ \\
\hline Aktinomyces spp. & $\begin{array}{l}\text { Gramfärbung } \\
\text { (grampositiv) }\end{array}$ & $\begin{array}{l}\text { lange Bebrü- } \\
\text { tungszeiten }\end{array}$ & nur experimentell & n.v. & n.v. & $\begin{array}{l}\text { Nachweis von Drusen } \\
\text { in der Histologie, hohe } \\
\text { Sensitivität und Spezi- } \\
\text { fität; seltener Erreger }\end{array}$ \\
\hline Nocardia spp. & $\begin{array}{l}\text { Gramfärbung, } \\
\text { Spezialfärbung } \\
\text { (Grocott-Gomori, } \\
\text { Silber) }\end{array}$ & $\begin{array}{l}\text { z. T. lange } \\
\text { Bebrütungs- } \\
\text { zeiten }\end{array}$ & nur experimentell & n.v. & n.v. & $\begin{array}{l}\text { partiell säurefest, Ver- } \\
\text { wechslung mit Myko- } \\
\text { bakterien; seltener Er- } \\
\text { reger; Abszessbildung }\end{array}$ \\
\hline
\end{tabular}

$10 \%$ angenommen [26]. Untersuchungen von CAPNETZ aus Deutschland widersprechen diesen älteren Studien und zeigen unter Anwendung einer validen PCR-Diagnostik eine sehr geringe Inzidenz von Chlamydieninfektionen (<1\%) [27]. Welche Bedeutung einer Besiedelung der Atemwege bei chronisch-obstruktiver Bronchitis durch C. pneumoniae zukommt, ist unklar. Mit dem Einsatz hochsensitiver PCR-Techniken wird in Abhängigkeit von Alter, vorbestehender Lungenfunktionsbeeinträchtigung oder akuter Exazerbation die Nachweisrate bei stabiler COPD erhöht; daraus lassen sich allerdings keine pathogenetischen, diagnostischen bzw. therapeutischen Schlussfolgerungen ziehen $[1,28]$.

\section{Diagnostische Verfahren \\ Kultur}

C. pneumoniae wird als obligat intrazellulärer Erreger ausschließlich in Zellkulturen eukaryontischer Zellen angezüchtet. Diese Methodik ist nur in wenigen Speziallaboratorien verfügbar, zudem fehlen verlässliche Angaben zu Sensitivität und Spezifität aufgrund erheblicher methodischer Unterschiede in den verwendeten Nachweisverfahren [29].

\section{Serologie}

Aus dem Serum können prinzipiell IgG-, IgM- und IgA-Antikörper bestimmt werden. Die Interpretation von Antikörper-Nachweisen ist jedoch aufgrund der hohen Prävalenz von IgG-Antikörpern bei Erwachsenen sowie dem Fehlen standardisierter Testsysteme schwierig. Hinzu kommt, dass IgM-Antikörper in aller Regel erst 2-3 Wochen nach Erkrankungsbeginn auftreten, IgG-Antikörper erreichen oft erst ca. 7 Wochen nach Erkrankungsbeginn hohe Titerstufen. Bei Reinfektionen ist eine IgMAntwort häufig nicht vorhanden und die IgG-Titer steigen innerhalb von $1-2$ Wochen an. Serologische Tests sind daher weniger für die Akutdiagnostik einer Chlamydieninfektion geeignet, eher für die retrospektive Bestätigung einer Diagnose oder für epidemiologische Studien [30]. IgA-Antikörper werden spät im Verlauf einer Infektion gebildet, sinken jedoch schneller als IgG-Antikörper wieder. Hohe IgA-Werte können bei einigen Patienten als Marker einer Reinfektion angesehen werden.

Der Mikroimmunfluoreszenztest zeigt wenig Kreuzreaktionen mit den beiden anderen Chlamydienspezies C. trachomatis und C. psittacii und ist der einzige etablierte Spezies-spezifische Antikörper-Test. Er ist jedoch technisch aufwendig, wenig standardisiert und von der Interpretation des Untersuchers abhängig. Ein IgM-Titer von $\geq 1: 16$ oder ein 4 -facher IgG-Titeranstieg in einem Serumpaar gilt als beweisend für eine akute Infektion [31]. Aufgrund persistierender postinfektiöser IgG- und IgM-Titer, zum Teil auch in hohen Konzentrationen, ist die Chlamydophila-pneumoniae-Serologie wegen falsch-positiver Ergebnisse in der Routinediagnostik nicht zu empfehlen [32]. In neuester Zeit werden zunehmend Westernblot-Techniken mit rekombinanten Chlamydophila-pneumoniae-Antigenen eingesetzt. Sie zeigen nach ersten Evaluierungen eine bessere Spezifität und Sensitivität, sind aber noch nicht abschließend zu bewerten.

\section{Amplifikationsverfahren}

Aufgrund der Einschränkung kultureller und serologischer Verfahren scheinen Nukleinsäure-Amplifikationsverfahren aus Atemwegsmaterialien geeignetere diagnostische Methoden für den Nachweis von C. pneumoniae zu sein. Angaben zu Sensitivität und Spezifität sind allerdings nicht möglich, da es keinen „Goldstandard“ als Vergleich gibt und die Variabilität unterschiedlicher NAT-Protokolle sehr hoch ist $[8,30]$. Die Centers for Disease Control der USA und das Laboratory Centre for Disease Control Canada geben Empfehlungen für die Standardisierung der NAT zum Nachweis von $C$. pneumoniae, doch bisher sind keine standardisierten Tests verfügbar, die sich für die Routinediagnostik eignen [30]. In Deutschland sind Ringversuche verfügbar, an denen die diagnostischen Labore teilnehmen sollten. 


\section{Empfehlung zur Diagnostik ( 0 Tab. 1)}

Eine Routinediagnostik einer Infektion durch C. pneumoniae wird bei Patienten mit ambulant erworbener Pneumonie, nosokomialer Pneumonie, akuter Bronchitis oder akuter Exazerbation einer chronischen Bronchitis nicht empfohlen.

\section{Chlamydophila psitacii \\ $\nabla$}

Als Reservoir von C. psittacii sind vor allem Vögel zu nennen. Daher sind meist Geflügelfarmen (Truthühner, Enten, Tauben) als Infektionsquelle für den Menschen von Bedeutung. Die atypischen Pneumonien sind häufig durch einen plötzlichen Beginn mit Schüttelfrost, hohem Fieber, trockenem Husten, Bradykardie und Kopfschmerzen gekennzeichnet. Der Schweregrad reicht von leichten Erkrankungen bis zu tödlich verlaufenden systemischen Infektionen, bei denen respiratorische Symptome im Vordergrund stehen.

\section{Empfehlung zur Diagnostik ( 0 Tab. 2)}

Die anamnestisch begründete Verdachtsdiagnose wird durch den Nachweis spezifischer Antikörper im Serum gestellt. Diese entwickeln sich erst im Verlauf der Infektion, sodass in der akuten Krankheitsphase mit einem negativen Ergebnis zu rechnen ist. Die Referenzmethode zum Nachweis Spezies-spezifischer Antikörper ist der Mikroimmunfluoreszenztest (MIF). Der kulturelle Nachweis ist wegen des Risikos schwerer Laborinfektionen nur in Laboratorien der Sicherheitsstufe III zugelassen. Der molekularbiologische Nachweis mittels PCR ist Spezies-spezifisch und potenziell hochsensitiv. Allerdings steht seine umfassende Validierung noch aus. Somit kann der Test noch nicht generell empfohlen werden.

\section{Kontaktadresse}

Konsiliarlabor für Chlamydien in Deutschland: Institut für Medizinische Mikrobiologie des Universitätsklinikums Jena: Ansprechpartner Prof. Dr. med. E. Straube, Dr. rer. nat. Jürgen Rödel Tel.: 03641-9-33905; juergen.roedel@med.uni-jena.de.

\section{Q-Fieber (Coxiella burnetii)}

C. burnetii ist ein kleines, unbewegliches, polymorphes, gramnegatives Bakterium aus der Familie der Rickettsiaceae. Er ist ein seltener Erreger einer interstitiellen Pneumonie, häufig begleitet von Muskelschmerzen und ausgeprägten Stirnkopfschmerzen. Seltener entwickelt sich eine Myokarditis bzw. Perikarditis oder Meningoenzephalitis. C. burnetii wird meist durch Inhalation infektiösen Staubes oder durch direkten Kontakt zu infizierten Tieren übertragen, weshalb der Anamneseerhebung eine herausragende Bedeutung bei der Diagnosestellung zukommt.

\section{Empfehlung zur Diagnostik ( 0 Tab. 2)}

Die Verdachtsdiagnose wird durch Antikörpernachweis labordiagnostisch gesichert. In Speziallaboratorien kann auch ein Erregernachweis mittels Zellkultur oder Nukleinsäure-Nachweis (PCR) erfolgen.

\section{Kontaktadresse}

Konsiliarlaboratorium für Coxiella burnetii, Institution: Landesgesundheitsamt Baden-Württemberg, Nordbahnhofstr. 135, 70191 Stuttgart, Ansprechpartner: Frau Dr. C. Wagner-Wiening, Tel.: 0711-904-39304; christiane.wagner-wiening@rps.bwl.de.

\section{Pseudomonas aeruginosa \\ $\nabla$}

P. aeruginosa sind gramnegative, nicht fermentierende Stäbchenbakterien (Non-Fermenter), die ubiquitär in der Umwelt (insbesondere in Feucht- und Nassbereichen) vorkommen und zum Teil schwere, lebensbedrohliche Infektionen bei immunsupprimierten, seltener bei immunkompetenten Patienten auslösen können. Da sie die Haut- und Schleimhäute von gesunden Menschen besiedeln, ist es im Einzelfall schwierig, zwischen einer Kolonisation und einer Infektion zu unterscheiden.

Aus pneumologischer Sicht haben Infektionen durch P. aerugino$s a$ in folgenden Situationen eine klinische Relevanz:

- Nosokomiale Pneumonie

P. aeruginosa gehören zu den häufigsten Erregern einer im Krankenhaus erworbenen Pneumonie in Abhängigkeit von den lokalen epidemiologischen und hygienischen Gegebenheiten, der Grunderkrankung des Patienten sowie dem Vorliegen bestimmter Risikofaktoren [6]. Hierzu zählen die Dauer des stationären Aufenthaltes vor Entwicklung der Pneumonie, invasive Beatmung und Beatmungsdauer sowie Vorbehandlung mit Antibiotika. Grunderkrankungen, die zu einer Infektion mit $P$. aeruginosa prädisponieren, sind vorwiegend strukturelle Lungenerkrankungen wie terminale chronischobstruktive Lungenerkrankung, zystische Fibrose oder Bronchiektasenerkrankung [33,34].

- Akute Exazerbation einer chronischen Bronchitis Untersuchungen zur bakteriellen Ätiologie der akuten Exazerbation einer chronisch-obstruktiven Bronchitis zeigen eine Abhängigkeit des Erregerspektrums von der vorbestehenden Lungenfunktionsbeeinträchtigung der stabilen COPD. P. aeruginosa und Enterobacteriaceae werden vermehrt bei einer $\mathrm{FEV}_{1}$ unter $50 \%$ isoliert, wobei zu beachten ist, dass bakterielle Erreger insgesamt nur in weniger als 50\% der Fälle nachgewiesen werden [35]. Ursächlich für den Erregershift dürften wiederholte Krankenhausaufenthalte und Antibiotikatherapien bzw. lokale Abwehrstörungen in der Bronchialschleimhaut („remodeling“) sein [36].

- Ambulant erworbene Pneumonie Nach den Daten von CAPNETZ sind Pseudomonaden als Erreger der ambulant erworbenen Pneumonie in Deutschland ausgesprochen selten [2]. Eine Berücksichtigung in der empirischen Therapie ist daher im Regelfall nicht erforderlich und wird nur bei schwer erkrankten Patienten mit Risikofaktoren, insbesondere bei struktureller Lungenerkrankung mit vorangegangener Antibiotikatherapie, vorausgegangenen Krankenhausaufenthalten oder bekannter Kolonisation empfohlen ( $\bullet$ Tab. $\mathbf{3}$ ).

Tab. 3 Risikofaktoren für eine Pseudomonas-aeruginosa-Infektion der unteren Atemwege bzw. bei Pneumonie.

sehr schwere chronisch-obstruktive Lungenerkrankungen, wie schwere COPD mit Antibiotika-Vortherapie oder vorausgegangener Hospitalisierung jeweils in den letzten drei Monaten

Bronchiektasen

Mukoviszidose

bekannte Kolonisation der Atemwege mit P. aeruginosa 


\section{Diagnostische Verfahren}

$P$. aeruginosa lassen sich grundsätzlich aus Materialien des Respirationstrakts, Blutkulturen und Pleuraflüssigkeit mittels mikrobiologischer Standardverfahren anzüchten. Der Nachweis aus Blutkulturen oder anderen Materialien aus primär sterilen Kompartimenten ist diagnostisch beweisend. Der Nachweis aus respiratorischem Material lässt hingegen per se keine Unterscheidung zwischen Infektion und Besiedelung zu [1]. Die Differenzierung zwischen Kolonisation und Infektion ist daher schwierig und bleibt trotz verschiedener Methoden zur Qualitätsverbesserung unbefriedigend, wie:

- Ermittlung einer Schwellenkonzentration (quantitative bzw. halbquantitative bakteriologische Aufarbeitung der Proben): Differenzielle diagnostische Schwellenkonzentration in Abhängigkeit vom Untersuchungsmaterial (Trachealsekret $\geq 106 / \mathrm{ml}$; bronchoalveoläre Lavage $\geq 10^{4} / \mathrm{ml}$ bzw. $\geq 10^{3} / \mathrm{ml}$ ) [6]

- mikroskopische Qualitätsbeurteilung der Proben: Anteil von Plattenepithelien ( $<10$ pro Gesichtsfeld bei $100 \times$ Vergrößerung) und Leukozyten ( $>25$ pro Gesichtsfeld) in den Proben [37]

- Nachweis von intrazellulären Erregern [6]

Bei beatmeten Patienten konnte bisher ein Vorteil bronchoskopisch gewonnener Proben gegenüber Trachealsekret nicht belegt werden, eine große Studie zu dieser Fragestellung schloss Patienten mit Risikofaktoren für $P$. aeruginosa aus [38].

Das Hauptproblem der Kultur liegt - neben der Dauer von 24-48 Stunden bis zum Vorliegen des Ergebnisses - in der niedrigen Sensitivität. Eine Resistenztestung ist obligat und wird vom mikrobiologischen Labor routinemäßig vorgenommen, da eine natürliche Resistenz gegenüber Penicillinen und Cephalosporinen besteht. Im Krankenhaus erworbene P. aeruginosa weisen darüber hinaus häufig zusätzliche Resistenzen gegenüber Carbapenemen oder Fluorchinolonen auf [39].

\section{Empfehlung zur Diagnostik ( 0 Tab. 2)}

Nosokomiale Pneumonie

Die Durchführung einer mikrobiologischen Diagnostik zum Nachweis von P. aeruginosa aus geeigneten Atemwegsmaterialien und weiteren Proben wie Blutkulturen oder Pleurapunktat wird bei Patienten mit nosokomialer Pneumonie empfohlen.

Die Sensitivitäten und Spezifitäten des Nachweises von Bakterien aus den (unsterilen) Untersuchungsproben des Respirationstraktes weisen große Streuungen auf. Die Genauigkeit der Ergebnisse wird entscheidend durch die Erfahrung der Intensivmediziner und des Untersuchungslabors, der Logistik im Untersuchungsablauf und der benutzten diagnostischen und klinischen Strategie bestimmt [6]. Der sorgfältigen klinischen Definition des Vorliegens einer nosokomialen Pneumonie kommt daher insbesondere bei invasiv beatmeten Patienten eine zentrale Rolle zu. Der Entwicklung einer Tubus-assoziierten Pneumonie geht in aller Regel eine Kolonisation der tiefen Atemwege mit dem Erreger voraus; daher sprechen sterile Kulturen aus Proben der unteren Atemwege ohne bestehende Antibiotikatherapie bzw. ohne vorherigen empirischen Wechsel einer Antibiotikabehandlung gegen das Vorliegen einer Pneumonie [40]. Eine Kombination aus klinischen und mikrobiologischen Parametern mit der Bestimmung von Biomarkern wie dem Procalcitonin im Serum vermag die Genauigkeit der Diagnostik einer Beatmungspneumonie signifikant zu verbessern [41]. Klinisch-mikrobiologische Parameter zur Definition einer Tubus-assoziierten Pneumonie können in einem einfachen und sensitiven Score (Clinical Pulmonary Infection Score CPIS) zusammengefasst werden:
- Temperatur (Scorepunkt 0: $\geq 36,5-\leq 38,4 ; 1: \geq 38,5-\leq 38,9$; $2: \geq 39,0$ oder $\leq 36,0)$

- Blutleukozyten $(0: \geq 4,0-\leq 11,0 ; 1:<4,0$ oder $>11,0 ; 2: 1$ und Segmentkernige $>0,5)$

- Trachealaspirat (0: kaum; 1 : vermehrt; 2 : vermehrt und purulent)

- Oxygenierung (PAO2/FIO2 mmHg; 0: > 240 oder ARDS; $2: \leq 240$ ohne ARDS)

- Pulmonale Infiltrate (0: keine; 1 : diffus; 2 : lokalisiert)

- Progression der pulmonalen Infiltrate ( $0:$ nein; $2: \mathrm{ja}$ )

- Kultur/Gramfärbung des Trachealaspirats (0: neg; 2: pos; Gramfärbung positiv: +1)

(Beurteilung durch Addition der Scorepunkte: Score <6: geringe Pneumonie-Wahrscheinlichkeit, Score $\geq 6$ : hohe PneumonieWahrscheinlichkeit) [42-44].

\section{Akute Exazerbation einer chronischen Bronchitis}

Die Kenntnis einer chronischen Besiedelung durch P. aeruginosa bei Patienten mit fortgeschrittener chronisch-obstruktiver Bronchitis kann in Analogie zu Patienten mit zystischer Fibrose die Auswahl geeigneter Antibiotika bei einer akuten Exazerbation steuern [1]. Bakteriologische Untersuchungen von Sputen bei COPD-Patienten mit fortgeschrittener Lungenfunktionseinschränkung sind daher zu empfehlen.

\section{Ambulant erworbene Pneumonie}

Ohne das Vorliegen entsprechender Risikofaktoren wie vorausgegangener Krankenhausaufenthalte, Antibiotikavortherapien, schwerer struktureller Lungenerkrankungen, Aspiration oder Malnutrition besteht bei Nachweis von P. aeruginosa aus respiratorischen Sekreten im Rahmen einer Routinediagnostik der ambulant erworbenen Pneumonie zunächst der Verdacht auf eine Kolonisation [45].

\section{Acinetobacter spp., Stenotrophomonas maltophilia und Burkholderia cepacia \\ $\nabla$}

Bei diesen Erregern handelt es sich um gramnegative, nicht fermentierende Stäbchenbakterien (Non-Fermenter) mit geringerer Virulenz als P. aeruginosa, denen insbesondere in der Ätiologie der späten nosokomialen Pneumonie ( $>5-7$ Tage nach stationärer Aufnahme) bei intubierten Patienten eine klinische Bedeutung zukommt. Zu berücksichtigen ist allerdings, dass diese Erreger - wie auch P. aeruginosa - in Abhängigkeit vom Vorliegen von Risikofaktoren wie antimikrobielle Vortherapie, vorhergehende Krankenhausaufenthalte, Prävalenz von resistenten Erregern in der Behandlungseinrichtung sowie Immunsuppression oder schwere hämatologische Grunderkrankungen auch bei Patienten mit früher nosokomialer Pneumonie und bei nicht intubierten Patienten als Pneumonierreger identifiziert werden können $[7,46,47]$. In den letzten Jahren wurde zunehmend über Resistenzen bei Non-Fermentern berichtet, die sich auf Fluorchinolone, Carbapeneme und Breitspektrum- $\beta$-Laktam-Antibiotika beziehen $[6,48]$.

\section{Diagnostische Methoden}

Non-Fermenter lassen sich grundsätzlich aus Materialien des Respirationstrakts, Blutkulturen und Pleuraflüssigkeit mittels mikrobiologischer Standardverfahren anzüchten, wobei der Nachweis aus Blutkulturen oder anderen Materialien aus primär sterilen Kompartimenten diagnostisch beweisend ist. Ihre Isolierung 
aus respiratorischem Material lässt hingegen per se keine Unterscheidung zwischen Infektion und Besiedelung zu [6].

\section{Empfehlung zur Diagnostik ( 0 Tab. 2)}

Insbesondere beim Vorliegen von Risikofaktoren für resistente Erreger sollte eine sorgfältige mikrobiologische Diagnostik vor Antibiotika-Therapieeinleitung bzw. -umstellung erfolgen und das mögliche Vorliegen von Non-Fermentern unter Berücksichtigung der lokalen Epidemiologie und Resistenzlage bei der Auswahl der antimikrobiellen Therapie berücksichtigt werden. Zur Befundinterpretation beim Nachweis dieser Erreger aus primär nicht-sterilen respiratorischen Materialien ist allerdings eine sorgfältige mikrobiologische bzw. klinische Definition der nosokomialen Pneumonie erforderlich.

Als spezifische Risikofaktoren für Pneumonien durch Acinetobacter spp. gelten:

- Aspiration im Krankenhaus

- ARDS

- vorausgegangener neurochirurgischer Eingriff

- Antibiotika-Vortherapie $[49,50]$

Für Pneumonien durch Stenotrophomonas maltophilia wurden als Risikofaktoren beschrieben:

- Aufenthalt auf einer Intensivstation

- Intubation

- Antibiotika-Vortherapie

- Neutropenie

- Diabetes mellitus bzw.

- hämatologische Systemerkrankungen [51,52]

\section{Actinomyces spp.}

$\nabla$

Actinomyces spp. sind fakultativ bis obligat anaerobe grampositive unbewegliche Stäbchen [53]. Der wichtigste Vertreter ist Actinomyces israeli. Ihr physiologisches Habitat ist die Mundhöhle. Schlechter Zahnstatus und parodontale Taschen begünstigen die Kolonisation im Oropharynx. Pathogenetisch spielen Mikro- bzw. Makroaspirationen für die Entwicklung einer pulmonalen Aktinomykose die wesentliche Rolle, begünstigt wird eine Infektion durch Mischinfektionen mit Sauerstoff-verbrauchenden Erregern auf dem Boden einer lokalen Gewebsschädigung [54]. Weitere klinische Manifestationen sind zervikofaziale und abdominelle Aktinomykosen.

Actinomyces spp. führen zu langsam progredienten Infektionen der Lunge, teilweise mit abszedierendem Verlauf, die gelegentlich zu Fistelbildungen, insbesondere kutanen Fisteln, neigen [55]. Radiomorphologisch imponieren sie nicht selten als intrapulmonale Raumforderungen, sodass differenzialdiagnostisch pulmonale Neoplasien ausgeschlossen werden müssen.

\section{Diagnostische Verfahren}

Die Diagnostik basiert auf dem histologischen Nachweis von Drusen im Gewebe, wobei nicht bei allen Actinomyces spp. diese Morphe anzutreffen ist. Im nach Gram gefärbten Untersuchungsmaterial sind fädige, schwach grampositve Elemente zu erkennen. Der kulturelle Nachweis spielt in der Praxis eine eher untergeordnete Bedeutung, da Bebrütungszeiten von bis zu 4 Wochen notwendig sind und diese nur auf spezielle Anforderung bei Verdacht durch den Kliniker im Labor eingehalten werden. Serologische Verfahren sind nicht etabliert, Nukleinsäure-Amplifikationsverfahren bisher nur experimentell.

\section{Empfehlung zur Diagnostik ( 0 Tab. 2)}

Bei klinischem Verdacht sollte der histologische Nachweis der Drusen im Gewebe angestrebt werden, da der Nachweis der Erreger aus respiratorischen Sekreten Ausdruck einer Kontamination aus dem Oropharynx sein kann und nur der histologische Nachweis von Strahlenpilzdrusen im Gewebe als pathognomonisch für diese Infektion gewertet wird.

\section{Nocardia spp. \\ $\nabla$}

Es gibt mehr als 20 verschiedene Nocardia-Spezies, wobei der $N$.asteroides-Komplex, bestehend unter anderem aus N. abscessus und N. asteroides, klinisch die größte Bedeutung besitzt. Es handelt sich um nicht Sporen-bildende, grampositive Stäbchen, die partiell säurefest sind und mikroskopisch Verzweigungen aufweisen. Sie sind in der Natur weit verbreitet, besonders bei biologischen Verwertungs- und Abbauprozessen im Erdboden. Als humane Krankheitserreger werden sie überwiegend bei abwehrgeschwächten Patienten mit T-Zell-Defekt nachgewiesen, wie Patienten nach Organtransplantation oder unter medikamentöser Immunsuppression. Sie kommen als sehr seltene Differenzialdiagnose auch beim abwehrgesunden Patienten mit einem chronischen Lungeninfiltrat in Betracht [55].

Eintrittspforte ist der Respirationstrakt, der Oropharynx gilt nicht als normales Habitat, sodass der Nachweis der Erreger aus oropharyngealem Sekret einen signifikanten Befund darstellt [56]. Klinisch manifestiert sich eine Nokardiose der Lunge als subakut bis chronisch verlaufende nekrotisierende Bronchopneumonie mit Neigung zur Abszedierung und hämatogener Metastasierung. Andere pulmonale Manifestationen sind Rundherde mit oder ohne Kavernenbildung sowie begleitende Pleuraergüsse [57]. Da eine Nokardiose häufig im Oberlappen auftritt, besteht die Notwendigkeit der differenzialdiagnostischen Abgrenzung zur Tuberkulose. Anreicherungen mit mikroskopischem Nachweis von säurefesten Stäbchen können irreführend sein, da Nocardia spp. schwach säurefest sind. Differenzialdiagnostisch sind ferner Infektionen durch Aspergillus spp. bzw. Toxoplasma gondii bei schwerem T-Zelldefekt sowie pulmonale Neoplasien auszuschließen.

\section{Diagnostische Verfahren}

Die Diagnostik basiert auf dem mikroskopischen und kulturellen Nachweis der Erreger aus Gewebe und respiratorischen Sekreten. Der kulturelle Nachweis erfolgt in der Regel nach Tagen, mitunter sind lange Bebrütungszeiten notwendig. Erfahrene Mikrobiologen können Nokardien mikroskopisch aus Sputum, Pleuraflüssigkeit, bronchoalaveolärer Lavage oder perkutanen Feinnadelaspiraten mit der Gramfärbung oder einer Spezialfärbung (Methamin-Silber oder Grocott-Gomori) rasch diagnostizieren. Es gibt Amplifikationsverfahren zum molekularbiologischen Nachweis der Erreger, diese sind aber nicht Teil der Standarddiagnostik einer Nokardiose [58].

\section{Empfehlung zur Diagnostik ( 0 Tab. 2)}

Es ist beim Transport der Proben darauf zu achten, dass diese nicht kühl gelagert werden dürfen [59]. Aufgrund der langen Bebrütungszeiten ist das mikrobiologische Untersuchungsinstitut auf den klinischen Verdacht hinzuweisen. 


\section{Kontaktadresse}

Konsiliarlaboratorium für Aktinomyzeten/Nokardien: Institut für Medizinische Mikrobiologie, Immunologie und Parasitologie Universitätsklinikum Bonn, Sigmund-Freud-Str. 25, 53105 Bonn, Ansprechpartner: Herr Prof. Dr. K. P. Schaal, Tel.: 0228/28711029, schaal@mibi03.meb.uni-bonn.de.

Der Nachweis seltener Erreger von Pneumonien (Tularämie, Keuchhusten, Klebsiellen) sowie der respiratorischen Viren und Pilze kann im Rahmen dieser Übersicht nicht abgehandelt werden. Es wird u. a. auf die Informationen der Robert-Koch-Instituts (www.rki.de) verwiesen.

\section{Zusammenfassung}

$\nabla$

Die Diagnostik von Infektionen der Atmwege sollte sich primär an den Empfehlungen und Leitlinien der Fachgesellschaften orientieren und die Grunderkrankung des Patienten, insbesondere das Vorliegen einer Abwehrschwäche, der Ort der Entstehung der Infektion (ambulant oder nosokomial) und die Art der Infektion (akute Exazerbation einer chronischen Bronchitis, ambulant erworbene oder nosokomiale Pneumonie) sowie die Schwere der klinischen Symptome und Befunde berücksichtigen. Im Vordergrund steht eine rasche Diagnostik, die bei Pneumonien den Beginn einer Antibiotika-Therapie keinesfalls verzögern darf. Im Zweifelsfall ist auf eine ätiologische Sicherung der Infektion zu verzichten bzw. diese im weiteren Verlauf anzustreben. Invasive Verfahren sollten erst bei therapierefraktärer Konstellation in Erwägung gezogen werden, da eine Erregersicherung in der Initialphase einer respiratorischen Infektion bei Berücksichtigung der Leitlinien und Empfehlungen nicht prognoserelevant ist.

\section{Interessenkonflikte}

Keine angegeben.

\section{Literatur}

1 Höffken G, Lorenz J, Kern W et al. S3-Leitlinie der Paul-Ehrlich-Gesellschaft für Chemotherapie, der Deutschen Gesellschaft für Pneumologie, der Deutschen Gesellschaft für Infektiologie und vom Kompetenznetzwerk CAPNETZ zu Epidemiologie, Diagnostik, antimikrobieller Therapie und Management von erwachsenen Patienten mit ambulant erworbenen tiefen Atemwegsinfektionen (akute Bronchitis, akute Exazerbation einer chronischen Bronchitis, Influenza und andere respiratorische Virusinfektionen) sowie ambulant erworbener Pneumonie. Pneumologie 2005; 59: 612-664

2 Welte T, Marre R, Suttorp N. Kompetenznetzwerk „Ambulant Erworbene Pneumonie“ (CAPNETZ). What is new in the treatment of community-acquired pneumonia? Med Klin 2006; 101: 313-320

3 Domínguez JA, Matas L, Manterola JM et al. Comparison of radioimmunoassay and enzyme immunoassay kits for detection of Legionella pneumophila serogroup 1 antigen in both concentrated and nonconcentrated urine samples. J Clin Microbiol 1997; 35: 1627-1629

4 Murdoch DR, Laing RT, Mills GD et al. Evaluation of a rapid immunochromatographic test for detection of Streptococcus pneumoniae antigen in urine samples from adults with community-acquired pneumonia. J Clin Microbiol 2001; 39: 3495 - 3498

5 Stralin K, Törnqvist E, Kaltoft MS et al. Etiologic diagnosis of adult bacterial pneumonia by culture and PCR applied to respiratory tract samples. J Clin Microbiol 2006; 44: 643-645

6 American Thoracic Society. Guidelines for the management of adults with hospital-acquired, ventilator-associated, and healthcare-associated pneumonia. Am J Respir Crit Care Med 2005; 171: 388-416

7 Mandell LA, Wunderink RG, Anzueto A et al. Infectious Diseases Society of America/American Thoracic Society consensus guidelines on the management of community-acquired pneumonia in adults. Clin Infect Dis 2007; 44: S27 - S72

8 Ieven $M$, Goossens $H$. Relevance of nucleic acid amplification techniques for diagnosis of respiratory tract infections in the clinical laboratory. Clin Microbiol Rev 1997; 10: 242 - 256

9 Smith CB, Golden CA, Kanner RE, Renzetti Jr. AD. Association of viral and Mycoplasma pneumoniae infections with acute respiratory illness in patients with chronic obstructive pulmonary diseases. Am Rev Respir Dis 1980; 121: $225-232$

10 Ruuskanen O, Nohynek H, Ziegler T et al. Pneumonia in childhood: Etiology and response to antimicrobial therapy. Eur J Clin Microb Infect Dis 1992; 11: $217-223$

11 Taylor-Robinson $D$. Infections due to species of mycoplasma and ureaplasma: An update. Clin Infect Dis 1996; 23: 671-682

12 Schmidt-Ioanas $M$, Bender $M$, Roth A et al. Early diagnosis of community-acquired pneumonia caused by Mycoplasma pneumoniae. Dtsch Med Wschr 2006; 131: 613-617

13 Watkins-Riedel T, Stanek G, Daxboeck F. Comparison of SeroMP IgA with four other commercial assays for serodiagnosis of mycoplasma pneumoniae pneumonia. Diagn Microbiol Infect Dis 2001; 40: 21 - 25

14 Beersma MFC, Dirven K, van Dam AP et al. Evaluation of 12 commercial tests and the complement fixation test for Mycoplasma pneumoniae specific immunoglobulin G (IgG) and IgM antibodies with PCR used as the "gold standard". J Clin Microbiol 2005; 43: 227-228

15 Templeton KE, Scheltinga SA, van den Eeden WC et al. Improved diagnosis of the etiology of community-acquired pneumonia with real-time polymerase chain reaction. Clin Infect Dis 2005; 41: 345 - 351

16 Helbig JH, Uldum SA, Luck PC et al. Detection of Legionella pneumophila antigen in urine samples by the BinaxNOW immunochromatographic assay and comparison with both Binax Legionella Urinary Enzyme Immunoassay (EIA) and Biotest Legionella Urin Antigen EIA. J Med Microbiol 2001; 50: $509-516$

17 Helbig JH, Uldum SA, Bernander S et al. Clinical utility of urinary antigen detection for diagnosis of community-acquired, travel-associated, and nosocomial legionnaires' disease. J Clin Microbiol 2003; 41: 838-840

18 Diederen BMW. Legionella spp. and Legionaires' disease. J Infect 2008; 56: $1-12$

19 Murdoch DR, Laing RT, Cook JM. The NOW S. pneumoniae urinary antigen test positivity rate 6 weeks after pneumonia onset and among patients with COPD. Clin Infect Dis 2003; 37: 153-154

20 Edelstein PH, Meyer RD, Finegold SM. Laboratory diagnosis of Legionnaires' disease. Am Rev Respir Dis 1980; 121: 317 - 327

21 Monforte R, Estruch R, Vidal J et al. Delayed seroconversion in Legionnaire's disease. Lancet 1988; 2: 513

22 Plouffe JF, File Jr. TM, Breiman RF et al. Reevaluation of the definition of Legionnaires' disease: use of the urinary antigen assay. Community Based Pneumonia Incidence Study Group. Clin Infect Dis 1995; 20: 1286 1291

23 Kallings I, Nordstrom $K$. The pattern of immunoglobulins with special reference to IgM in Legionnaires' disease patients during a 2 year follow-up period. Zentralbl Bakteriol Mikrobiol Hyg [A] 1983; 255: 27 32

24 Musso D, Raoult D. Serological cross-reactions between Coxiella burnetii and Legionella micdadei. Clin Diagn Lab Immunol 1997; 4: 208 212

25 Steinhoff $D$, Lode $H$, Ruckdeschel $G$ et al. Chlamydia pneumoniae as a cause of community-aquired pneumonia in hospitalized patients in Berlin. Clin Clin Dis 1996; 22: 958 - 964

26 Kauppinen M, Saikku P. Pneumonia due to Chlamydia pneumoniae: prevalence, clinical feature, diagnosis, and treatment. Clin Infect Dis 1995; 21 (Suppl. 3): S244-S254

27 Wellinghausen $N$, Straube E, Freidank $H$ et al. Low prevalence of Chlamydia pneumoniae in adults with community-acquired pneumonia. Int J Med Microbiol 2006; 296: 485 - 491

28 Blasi F, Damato S, Cosentini R, Tarsia P, Raccanelli R, Centanni S, Allegra L and the Chlamydia InterAction with COPD (CIAC) Study Group. Chlamydia pneumoniae and chronic bronchitis: association with severity and bacterial clearance following treatment. Thorax 2002; 57: 672-676

29 Tompkins LS, Schachter J, Boman J. Collaborative multidisciplinary workshop report: detection, culture, serology, and antimicrobial susceptibility testing of Chlamydia pneumoniae. J Infect Dis 2000; 181 (Suppl. 3): S460 - S461

30 Dowell SF, Peeling RW, Boman J, Carline GM, Fields BS, Guarner J, Hammerschlag MR, Jackson LA, Kuo C-C, Maass M, Messmer TO, Talkington DF, Tondella ML, Zaki SR and the C pneumoniae workshop participants. 
Standardizing Chlamydia pneumoniae assays: Recommendations for the Centers for Disease Control and Prevention (USA) and the Laboratory Centre for Disease (Canada). Clin Infect Dis 2001; 33: 492 - 502

31 Dowell J, Pitkethly M, Bain J, Martin S. A randomised controlled trial of delayed antibiotic prescribing as a strategy for managing uncomplicated respiratory tract infection in primary care. Br J Gen Pract 2001; 51: 200-205

32 Peeling RW, Wang S-P, Grayston JT et al. Chlamydia pneumoniae serology: Interlaboratory variation in microimmunfluorescence assay results. J Infect Dis 2000; 181: S426-S429

33 Trouillet JL, Chastre J, Vuagnat A et al. Ventilator-associated pneumonia caused by potentially drug-resistant bacteria. Am J Respir Crit Care Med 1998; 157: 531 - 539

34 Rello J, Ausina V, Ricart M et al. Risk factors for infections by Pseudomonas aeruginosa in patients with ventilator-associated pneumonia. Intensive Care Med 1994; 20: 193-198

35 Eller J, Ede A, Schaberg T et al. Infective exacerbations of chronic obstructive pulmonary disease. Relation between bacteriologic etiology and lung function. Chest 1998; 113: $1542-1548$

36 Wilkinson TM, Patel IS, Wilks $M$ et al. Airway bronchial load and Fev1 decline in patients with chronic obstructive pulmonary disease. Am J Respir Crit Care Med 2003; 167: 1090-1095

37 Welte T. Die nosokomiale Pneumonie. Intensivmedizin 2006; 43: 301 309

38 Canadian Critical Care Trials Group. A randomized trial of diagnostic techniques for ventilator-associated pneumonia. N Engl J Med. 2006; 355: $2619-2630$

39 Livermore DM. Multiple mechanisms of antimicrobial resistance in Pseudomonas aeruginosa: Our worst nightmare? Clin Infect Dis 2002; 34: $634-640$

40 Kirtland SH, Corley DE, Winterbauer RH et al. The diagnosis of ventilator-associated pneumonia: a comparison of histologic, microbiologic, and clinical critera. Chest 1997; 112: 445-457

41 Ramirez P, Garcia MA, Ferrer $M$ et al. Sequential measurements of procalcitonin levels in diagnosing ventilator-associated pneumonia. Eur Respir J. 2008; 31: 356-362

42 Pugin J, Auckenthaler R, Mili N et al. Diagnosis of ventilator-associated pneumonia by bacteriologic analysis of bronchoscopic and nonbronchoscopic „blind“ bronchoalveolar lavage fluid. Am Rev Respir Dis 1991; 143: 1121 - 1129

43 Fartoukh M, Maitre B, Honoré $S$ et al. Diagnosing pneumonia during mechanical ventilation. Am J Respir Crit Care Med 2003; 168: 173179

44 Singh N, Rogers P, Atwood CW et al. Short-course empiric antibiotic therapy for patients with pulmonary infiltrates in the intensive care unit. A proposed solution for indiscriminate antibiotic prescription. Am J Respir Crit Care Med 2001; 164: 172-173

45 Arancibia F, Bauer TT, Ewig S et al. Community-acquired Pneumonia caused by Gram-negative bacteria: Incidence and risk and prognosis. Arch Intern Med 2002; 162: 1849-1858
46 Ibrahim EH, Ward S, Sherman G, Kollef MH. A comparative analysis of patients with early-onset vs late-onset nosocomial pneumonia in the ICU setting. Chest 2000; 117: 1434-1442

47 Weber DJ, Rutala WA, Sickbert-Bennett EE et al. Microbiology of ventilator-associated pneumonia compared with that of hospital-acquired pneumonia. Infect Control Hosp Epidemiol 2007; 28: 825-831

48 Namis N, Samiian L, Nino D et al. Incidence and susceptibility of pathogenic bacteria vary between intensive care units within a single hospital: implications for empiric antibiotic strategies. J Trauma 2000; 49: $638-646$

49 Baraibar J, Correa H, Mariscal D et al. Risk factors for infection by Acinetobacter baumannii in intubated patients with nosocomial pneumonia. Chest 1997; 112: $1050-1054$

50 Falagas ME, Rafailidis PI. Attributable mortality of Acinetobacter baumannii: no longer a controversial issue. Crit Care 2007; 134: 1-3

51 Aisenberg G, Rolston KV, Dickey BP et al. Stenotrophomonas maltophilia in cancer patients without traditional risk factors for infection, 19972004. Eur J Clin Microbiol Infect Dis 2007; 26: 13-20

52 Fedler KA, Biedenbach DJ, Jones RN. Assessment of pathogen frequency and resistance patterns among pediatric patient isolates: report from the 2004 SENTRY Antimicrobial Surveillance Program on 3 continents. Diagn Microbiol Infect Dis 2006; 56: 427-436

53 Goetz MB, Finegold SM. Actinomycosis. In: Murray FJ, Nadel JA, eds. Textbook of Respiratory Medicine. 3rd edition Vol 1. Philadelphia, PA: W.B. Saunders Company, 2000: 1020-1022

54 Russo TA. Actinomycosis. In: Kasper DL, Braunwald E, Fauci AS, Hrsg Harrison's Principles of Internal Medicine. 16th ed. Vol.1. New York, NY: McGraw-Hill, 2005: 937-939

55 Yildiz O, Doganay M. Actinomycosis and nocarida pulmonary infections. Curr Opin Pulm Med 2006; 12: 228-234

56 Raich RA, Casey F, Hall WH. Pulmonary and cutaneous nocardiosis. The significance of the laboratory isolation of Nocardia. Am Rev Respir Dis 1961; 83: 505- 509

57 Lederman ER, Crum NF. A case series and focused review of nocardiosis. Medicine 2004; 83: 300 - 313

58 Tatt KM, Shieh WJ, Phillips S, Augenbraun M, Rao C, Zaki SR. Molecular diagnosis of Nocardia farcina from a cerebral abscess. Hum Pathol 2006; 37: 1117-1121

59 Marre R, Mertens T, Trautmann M, Zimmerli W. Klinische Infektiologie. 2. Auflage. München: Urban und Fischer, 2008: 1142

\section{Bisher erschienene Beiträge dieser Serie}

1 Strassburg A et al. Infektionsdiagnostik in der Pneumologie. Pneumologie 2008; 62: 730-743

2 Rohde G et al. Nachweis von Atemwegsviren - wie, warum, wann und wo? Pneumologie 2009; 63: 14-22

3 Gillissen A et al. Biomarker bei infektiösen und nicht infektiösen Lungenerkrankungen außer Malignome. Pneumologie 2009; 63: 439-450 